\title{
Single-color photoassociative ionization of ultracold sodium: The region from 0 to $-5 \mathrm{GHz}$
}

\author{
A. Amelink, ${ }^{1}$ K. M. Jones, ${ }^{2}$ P. D. Lett, ${ }^{2}$ P. van der Straten, ${ }^{1}$ and H. G. M. Heideman ${ }^{1}$ \\ ${ }^{1}$ Debye Institute, Department of Atomic and Interface Physics, Utrecht University, P.O. Box 80000, 3508 TA \\ Utrecht, The Netherlands \\ ${ }^{2}$ Atomic Physics Division, National Institute of Standards and Technology, Gaithersburg, Maryland 20899-8424
}

(Received 28 October 1999; revised manuscript received 24 February 2000; published 13 June 2000)

\begin{abstract}
We have measured the rate of production of $\mathrm{Na}_{2}{ }^{+}$ions in collisions of ultracold $\mathrm{Na}$ atoms held in a magneto-optical trap (MOT) as a function of probe laser detuning using a single-color probe beam. The ion rate is measured with the atoms mainly in the $3 S(f=2)$ level ("bright MOT") or the $3 S(f=1)$ level ("dark MOT'). Using recent experimental information about the doubly excited autoionizing states of $\mathrm{Na}_{2}$, we find that the large structures in the first $5 \mathrm{GHz}$ red of the atomic transition frequency are due to a doubly resonant excitation process with levels in the $0_{g}^{-}$potential (asymptotically connected to $3 S_{1 / 2}+3 P_{3 / 2}$ ) as the intermediate states, and levels in the $0_{u}^{-}$and $1_{u}$ potentials (asymptotically connected to $3 P_{3 / 2}+3 P_{3 / 2}$ ) as the final autoionizing states. We can account for nearly all of the observed structure in this region of the spectra for both the bright and dark MOT.
\end{abstract}

PACS number(s): 32.80.Pj, 33.20.-t, 34.50.Gb

\section{INTRODUCTION}

The study of collisions between cold atoms $(T \approx 1 \mathrm{mK})$ has been made possible by the introduction of laser cooling and trapping techniques. When two atoms collide in the presence of light, the atoms may combine to produce a bound, electronically excited molecule. This photoassociation process may be detected as a decrease in the fluorescence of the trapped atomic sample [1]. In the particular case of sodium, two atoms colliding in the presence of light tuned to the red of the atomic $3 S \rightarrow 3 P_{3 / 2}$ transition can photoassociate and then absorb a second photon of the same color to produce an $\mathrm{Na}_{2}{ }^{+}$ion. Early experiments studied this photoassociative ionization (PAI) rate for atoms held in an all-optical trap [2]. Resonant structures were observed in the ion signal as the trap lasers were detuned from the atomic transition by up to $5 \mathrm{GHz}$. Improved experiments using a magneto-optical trap (MOT) and a separate probe laser to produce the ions have shown that photoassociative ionization signals with a single color probe laser can be observed far red of the first $5 \mathrm{GHz}$ of detuning $[1,3,4]$. Figure 1 shows such a spectrum. Still, a clear distinction can be made between the spectrum measured in the region between 0 and $-5 \mathrm{GHz}$ ("the first 5 $\mathrm{GHz}$ ') and the spectrum measured in the region below $-5 \mathrm{GHz}$.

Below the first $5 \mathrm{GHz}$, regularly spaced ion peaks are observed. Identification of these peaks is based on their regular vibrational spacing and hyperfine structure (see Ref. [3] for a more extensive discussion of the spectrum in this region). The spectrum in this region is dominated by photoassociation at long range $\left(R \geqslant 140 a_{0}\right)$ to vibrational levels of the singly excited $1_{g}\left(3 S+3 P_{3 / 2}\right)$ potential. This is followed by a second transition to an ionization continuum at relatively short range. In Fig. 2 this mechanism is shown schematically. The first step (photoassociation) is represented by a solid arrow from the ground state to a bound level of the $1_{g}$ state, the second step (photoionization) by a solid arrow from this $1_{g}$ bound state to the ionization continuum at short internuclear distance. In addition to this $1_{g}$ progression, similar ionization signals belonging to a $0_{u}^{+}$state have been observed [3].

Trap loss signals indicating photoassociation to a $0_{g}^{-}$state are also observed in this spectral region, but no corresponding ion signal is observed. The $0_{g}^{-}$state is a purely longrange state (see Fig. 4). Because vibrational levels in this potential have inner turning points of $R>50 a_{0}$, the shortrange ionization mechanism which gives rise to the ion signals for the $1_{g}$ levels does not occur for the $0_{g}^{-}$levels [5].

In this paper we investigate the large, irregularly spaced features which appear in the first $5 \mathrm{GHz}$ of the spectrum. Plausible explanations for this new structure might invoke some change in the character of either the photoassociation or ionization steps or both. From the one-color spectrum (Fig. 1) alone it is impossible to distinguish between these possibilities. By doing auxiliary two-color experiments in which we use a separate, independently tunable laser for each step, we can disentangle the contributions of the photoassociation and ionization steps. We will show that we can

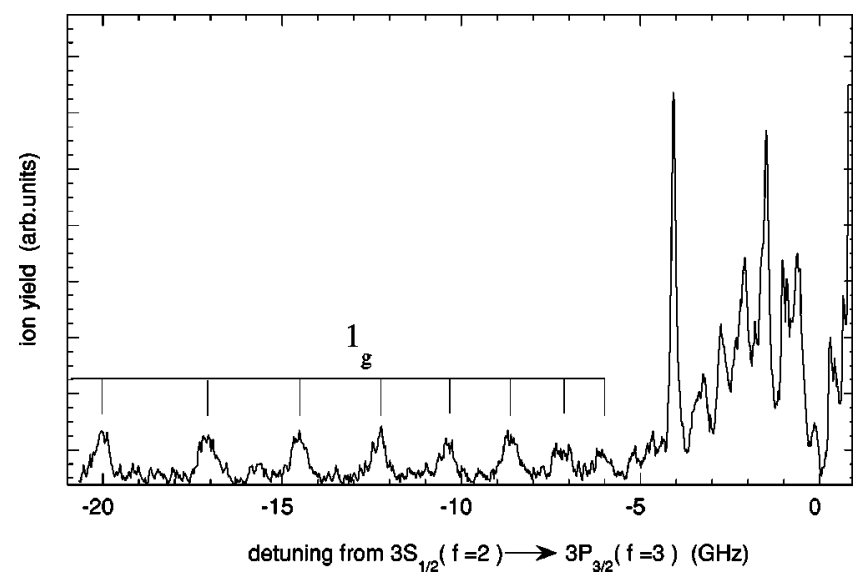

FIG. 1. Signal of $\mathrm{Na}_{2}{ }^{+}$ions as a function of probe laser detuning relative to the atomic transition frequency $3 S_{1 / 2}(f=2)$ $\rightarrow 3 P_{3 / 2}(f=3)$, showing the sudden increase in signal for small detunings. 


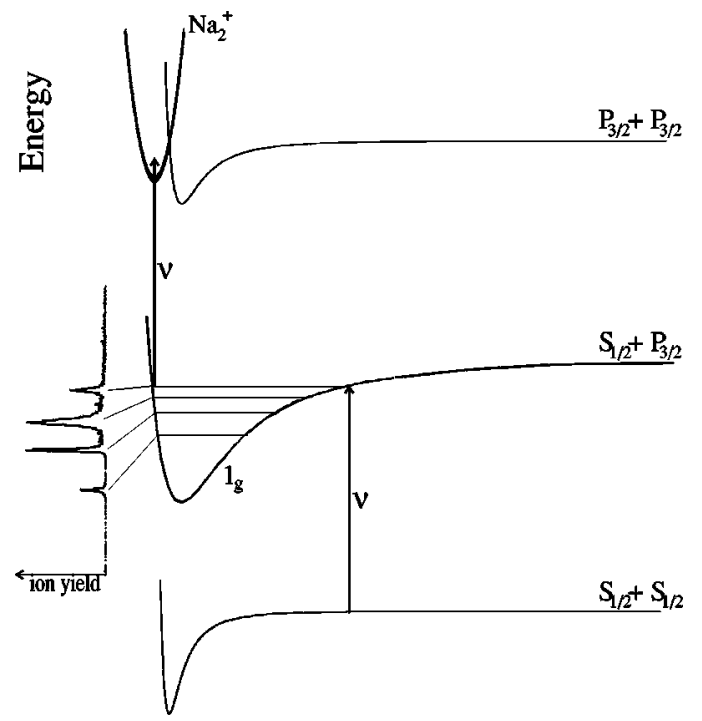

Internuclear distance

FIG. 2. Schematic diagram showing an ionization mechanism in the cold collisions of sodium atoms. The first step of the two-step process is the photoassociation of two slowly colliding atoms into a singly excited bound molecular state by the absorption of a red detuned photon. The second step is direct photoionization at small internuclear distances. When the probe laser is scanned, an ion signal is observed only when the probe laser is resonant with a transition from the ground state to a singly excited bound molecular state.

explain essentially all the structure in the first $5 \mathrm{GHz}$ of the one-color spectrum as the result of photoassociation to vibrational levels in the purely long-range $0_{g}^{-}$potential followed by excitation to a bound, autoionizing level of a doubly excited molecular potential connecting to the $3 P_{3 / 2}+3 P_{3 / 2}$ asymptote. This process is schematically illustrated in Fig. 3. The change in character of the signal in the first $5 \mathrm{GHz}$ is thus a result of the opening of a new ionization pathway which permits photoassociation to the $0_{g}^{-}$intermediate state to contribute to the ion signal. In order for a peak to appear in the single-color spectrum, it is necessary that there be an accidental coincidence between the energy of the photoassociation step and the ionization step. This leads to the irregular appearance of the signals.

This mechanism is essentially that proposed by Heather and Julienne (HJ) [6] in response to the original experimental observation of ionization in the detuned optical trap [2]. At the time, the nature of the doubly excited states was unknown and so a model doubly excited $1_{u}$ potential was adjusted to give a cutoff in agreement with the observed spectrum. Recently, we have used two-color experiments to investigate the autoionizing states that are important for this process [7]. We find that there are in fact two doubly excited autoionizing potentials (one of $1_{u}$ and one of $0_{u}^{-}$symmetry) which are important, neither of which has the shallow depth proposed by HJ. The cutoff in the spectrum is instead due to a rapidly decreasing Franck-Condon overlap between the $0_{g}^{-}$ state and autoionizing states bound by more than $10 \mathrm{GHz}$. Combining these new data with other two-color experiments which have studied the $0_{g}^{-}$state $[8,9]$, we are able to repro-

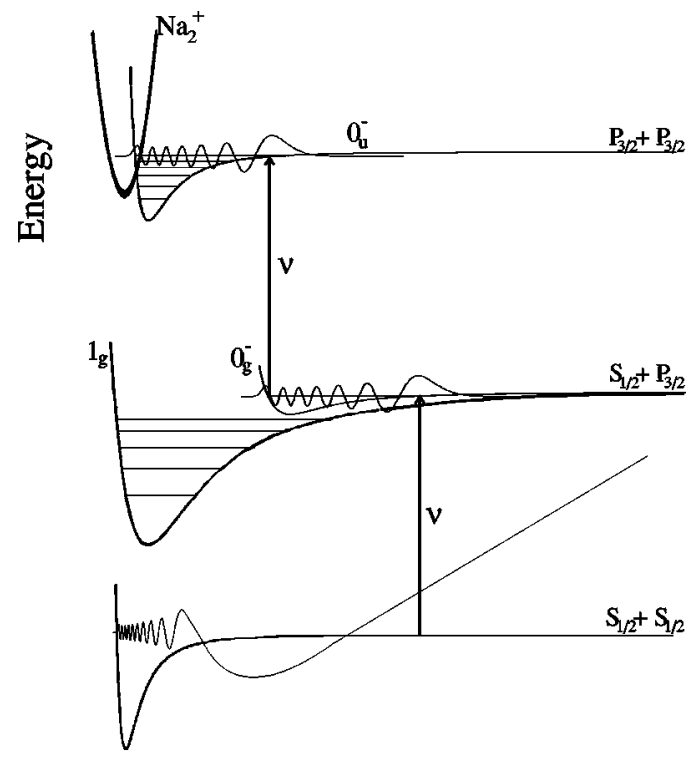

Internuclear distance

FIG. 3. Schematic representation of the doubly resonant ionization process responsible for the large structures in the first $5 \mathrm{GHz}$ of the single-color ionization spectrum. In addition to the $0_{u}^{-}$state, there is also a doubly excited $1_{u}$ state which autoionizes at short internuclear distances. Only the long-range parts $\left(R>12 a_{0}\right)$ of these two potentials are known experimentally; the potential drawn here is meant only as an illustration.

duce, from measured two-color spectra, the main features observed in the single-color spectrum in the first $5 \mathrm{GHz}$. Spectra taken with most of the atoms initially in either the lower, $3 S(f=1)$, or the upper, $3 S(f=2)$, atomic hyperfine state show differences that can also be accounted for by our model.

\section{REVIEW OF THE POTENTIALS INVOLVED}

\section{A. Ground states}

The molecular ground-state interaction at long range is the attractive $C_{6} / R^{6}$ van der Waals interaction, with $C_{6}=$ -1539 a.u. for $\mathrm{Na}_{2}$ [10]. At an internuclear distance of $100 a_{0}$, the van der Waals interaction is only $10 \mathrm{MHz}$ and the ground-state potential is considered to be flat over the range of internuclear distances relevant to our experiments. Including the hyperfine interaction in the ground-state of $\mathrm{Na}$, there are three ground state potentials: we distinguish the $2+2$, the $1+2$, and the $1+1$ molecular hyperfine ground states, where we have indicated $f_{a}+f_{b}$ of the two atoms. These states are split in energy by the atomic ground-state hyperfine splitting $\Delta E_{\mathrm{hfs}}=1.772 \mathrm{GHz}$.

\section{B. Singly excited states}

If there were no atomic fine-structure splitting, then the long-range behavior of the states at the $3 S+3 P$ dissociation limit would be determined by the purely $C_{3} / R^{3}$ resonant dipole interaction. The $C_{3}$ depends on the relative orientation of the $3 S$ and the $3 P$ atoms, i.e., on the symmetry of the 


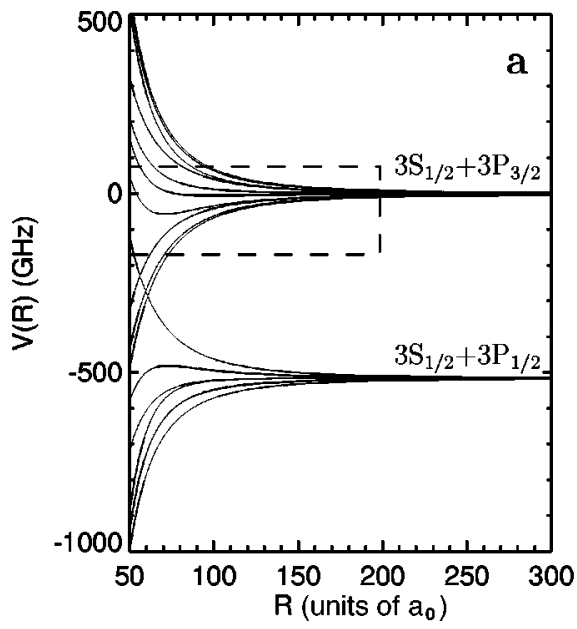

molecular state. The competition between the fine structure and resonant dipole interactions produces potentials which are not simply $1 / R^{3}$, as discussed in detail by Movre and Pichler [11]. At long range, the dipole-dipole interaction is much stronger than the van der Waals interaction: at an internuclear distance of $100 a_{0}$, the dipole-dipole interaction is $66 \mathrm{GHz}$ using a typical $C_{3}$ value of 10 a.u. With this $C_{3}$ value, the potential becomes $5 \mathrm{GHz}$ deep at an internuclear distance of $\approx 240 a_{0}$.

Figure 4 shows a plot of the calculated $\mathrm{Na}_{2}$ Hund's case (c) potentials connecting asymptotically to the $3 S_{1 / 2}$ $+3 P_{1 / 2,3 / 2}$ dissociation limits. In our experiment, the probe laser which excites a pair of atoms to a bound molecular state was tuned to the red (blue) of the $3 S_{1 / 2} \rightarrow 3 P_{3 / 2}\left(3 P_{1 / 2}\right)$ atomic transition frequency. Therefore, we only need to consider those molecular potentials which are attractive and asymptotically connecting to the $3 S_{1 / 2}+3 P_{3 / 2}$ dissociation limit. Figure 4 shows that five molecular symmetries fulfill the necessary requirements: $1_{u}, 0_{g}^{-}, 2_{u}, 1_{g}$, and $0_{u}^{+}$. The $1_{u}$ potential is a purely long-range potential with a well depth of only $\approx 5 \mathrm{GHz}$, while the $0_{g}^{-}$potential is also purely long range with a well depth of $\approx 57 \mathrm{GHz}[9,12,13]$. These two states, at long range attractive, become repulsive at relatively large internuclear distances $\left(R>50 a_{0}\right)$ due to avoided crossings with repulsive states connecting to the $3 S_{1 / 2}+3 P_{1 / 2}$ dissociation limit. Bound levels in these potentials have inner turning points at large internuclear distances and their properties can be calculated to high precision from the atomic properties of $\mathrm{Na}$. The $2_{u}, 1_{g}$, and $0_{u}^{+}$potentials remain attractive to much shorter internuclear distances. Transitions from the ground state to the $2_{u}$ state are dipole forbidden.

\section{Doubly excited states}

The dominant interaction term for the states connecting to the $3 P+3 P$ dissociation limit is the $C_{5} / R^{5}$ quadrupolequadrupole interaction. Curve crossings from other asymptotes complicate the picture [15] so that a simple MovrePichler type of analysis is not sufficient. However, we have shown [7] that the two autoionizing states important for the present purposes are consistent with a $C_{5} / R^{5}$ interaction with $C_{5}=-254$ a.u. At long range the quadrupolequadrupole interaction is much weaker than the dipole-dipole
FIG. 4. Calculated Hund's case (c) potential curves for $\mathrm{Na}_{2}$ connected to the $3 S_{1 / 2}+3 P_{1 / 2,3 / 2}$ dissociation limits. In (a) all the molecular potentials are shown including fine-structure (the finestructure splitting is $\Delta_{\mathrm{fs}}$ $=515.52 \mathrm{GHz})$, and in (b) all the curves connecting to the $3 S_{1 / 2}$ $+3 P_{3 / 2}$ dissociation limit are shown.

\section{EXPERIMENT}

The experiments are performed in both a bright and a dark MOT $[16,17]$. In both types of MOT, the trapping laser drives the $3{ }^{2} S_{1 / 2}(f=2) \rightarrow 3{ }^{2} P_{3 / 2}(f=3)$ atomic transition (type-I cooling). Occasionally atoms are excited to the $3^{2} P_{3 / 2}(f=2)$ state, which can decay to the $3{ }^{2} S_{1 / 2}(f=1)$ ground state. A "repumper' laser is introduced by leading a fraction of the trapping laser light through a $1.7 \mathrm{GHz}$ acousto-optic modulator. In the case of the bright MOT, the entire trapping volume is covered with repumping light, which pumps most of the atoms into the $f=2$ hyperfine ground state. In the case of the dark MOT, a dark spot in the middle of the repumping beams leaves the central volume of the trap free of the repumping light. As a consequence, most of the trapped atoms are optically pumped into the $f=1$ ground state.

The trapping beams are turned on and off at a $100 \mathrm{kHz}$ rate with a duty cycle of $50 \%$. During the off periods of the trapping laser, a variable-frequency probe laser is introduced into the MOT. Low intensities are used to avoid power broadening. The relative frequency of the probe laser is determined from a calibrated $300 \mathrm{MHz}$ free spectral range confocal etalon which is locked to the saturated absorption spectrum of $\mathrm{Na}$. The absolute frequency is determined from a simultaneously recorded $I_{2}$ absorption spectrum. Molecular ions created during the probe periods are collected and counted with a channel electron multiplier. Similar experiments are described in greater detail in $[3,14]$. 


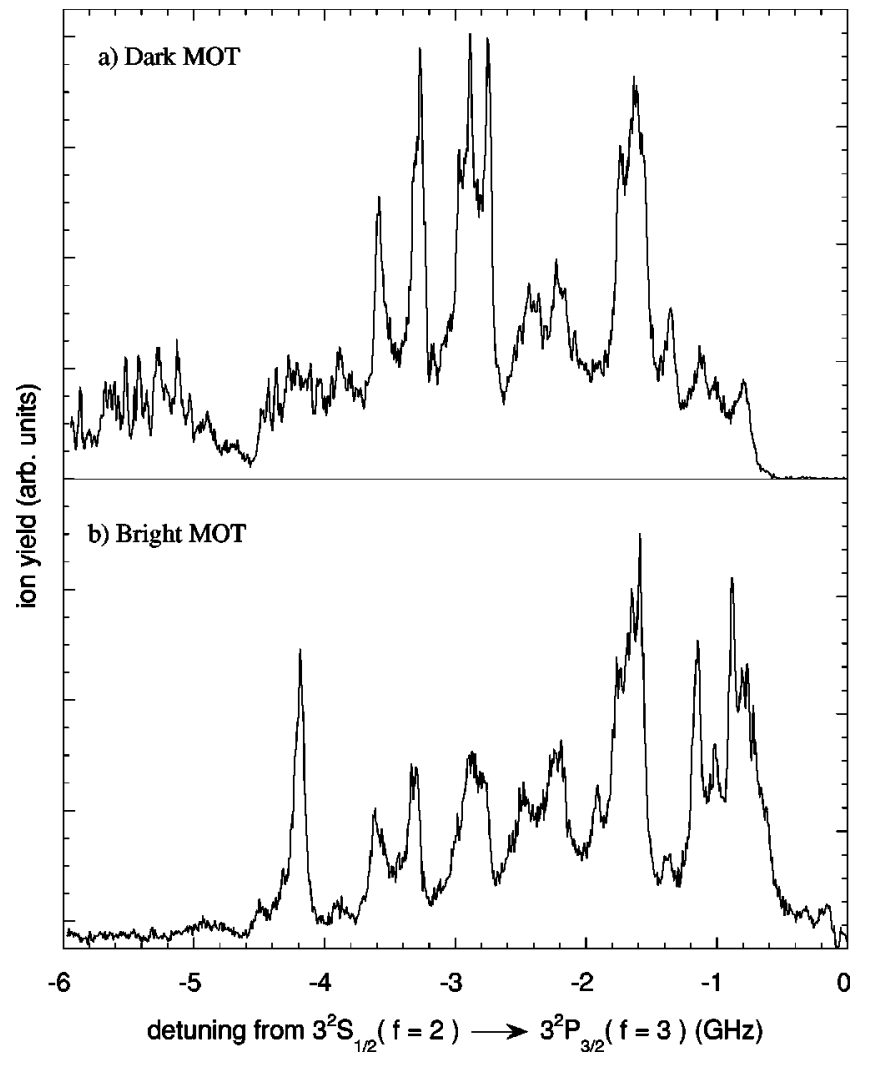

FIG. 5. Ion yield as a function of probe laser detuning in (a) a dark MOT and (b) a bright MOT, close to the atomic transition frequency.

\section{RESULTS}

\section{A. Single-color PAI}

Our single-color PAI measurements for small detunings in the bright and dark MOT are shown in Fig. 5. Multiple low-power scans were averaged to increase the signal-tonoise ratio. The noise level is indicated by the most rapid fluctuations, as exemplified by the region between -5 and $-6 \mathrm{GHz}$ detuning in Fig. 5(b). The noise is slightly larger at larger signal levels, being mostly due to fluctuations in the loading of the trap. Most of the structure, for example, between -5 and $-6 \mathrm{GHz}$ in Fig. 5(a) is not noise but represents $\approx 10$ narrow peaks. A comparison between the bright and dark MOT spectra shows that the ion signal in the first 5 $\mathrm{GHz}$ depends on the relative populations of the two atomic hyperfine ground states. Most of the atoms in the bright MOT are in the $f=2$ hyperfine ground state, whereas in the dark MOT the $f=1$ ground state is mostly populated. In the bright MOT most of the collisions are thus between two atoms in the $f=2$ ground hyperfine state $(2+2$ collisions), fewer collisions are with one atom in the $f=2$ and the other in the $f=1$ state $(1+2$ collisions), while the number of collisions between two atoms in the $f=1$ state $(1+1$ collisions $)$ will be relatively small. In the dark MOT it is opposite: there will be a relatively large number of $1+1$ collisions, fewer $1+2$ collisions, and even fewer $2+2$ collisions. The different ground-state pairs are split in energy by $1.77 \mathrm{GHz}$. Transitions from the different combinations of colliding ground- state atoms to a given intermediate level will thus be shifted in frequency. For instance, a transition to a given intermediate level starting from a $1+1$ collision will be shifted 1.77 $\mathrm{GHz}$ to the blue with respect to a transition to the same intermediate level starting from a $1+2$ collision. In addition, the wave-function overlap with the excited states is different for the three ground-state pairs [12]; possibly their transition dipole moments are different as well. For these reasons it is expected that the PAI spectrum will be different for each ground-state pair. The PAI spectra measured in both the bright and dark MOTs are the sum of spectra from the three ground-state pairs, but with different weighting factors. Thus the differences between the bright and dark MOT spectra are most likely the result of the difference in atomic ground-state populations in the two MOTs. This will be investigated in more detail in Sec. V.

\section{B. Two-color probe of the ionization step}

In this section we will set out to demonstrate experimentally that the singly excited $0_{g}^{-}$potential is the only intermediate state responsible for the structure in the first $5 \mathrm{GHz}$. For this purpose we have performed two-color experiments $[7,14]$ with the photoassociating (PA) laser fixed on a vibrational level in each of the three singly excited potentials that might be involved in the ionization process (the $1_{g}, 0_{g}^{-}$, and $0_{u}^{+}$dissociating to $3 S+3 P_{3 / 2}$ [3]). The binding energies of the chosen levels were all $\approx-30 \mathrm{GHz}$. At this detuning the three states are clearly resolved. A second, photoionizing (PI) laser was scanned to produce the spectra shown in Fig. 6.

Bound doubly excited states are observed only with the PA laser fixed on the $0_{g}^{-}$state. Longer scans than these do not show any bound states below $-10 \mathrm{GHz}$. There is a small, structureless background ionization level present across the entire spectrum obtained with the PA laser fixed on the $1_{g}$ and $0_{u}^{+}$intermediate states. This background signal is possibly due to a single-color ionization process such as that indicated in Fig. 2, where the second step is made with a photon from either the PA or the PI laser, independent of the frequency of the PI laser. This direct photoionization process from bound levels in the $0_{u}^{+}$and $1_{g}$ states is also possible for probe laser detunings less than $5 \mathrm{GHz}$ below atomic resonance, but it does not result in additional structure since the photoionization step is a bound $\rightarrow$ free transition into the ionization continuum. Only if the second step involves a bound $\rightarrow$ bound transition is additional structure expected to arise. The fact that bound doubly excited levels are only observed with the $0_{g}^{-}$state as the intermediate state therefore implies that it is the only intermediate state involved in the change in character of the single-color spectrum near $5 \mathrm{GHz}$ detuning.

The fact that no bound states are observed with the PA laser fixed on the chosen vibrational levels of the $1_{g}$ and $0_{u}^{+}$ potentials can be understood by considering the FranckCondon wave-function overlap of these levels with the doubly-excited states. The outer turning points of the leastbound rovibrational levels of the doubly-excited states are 


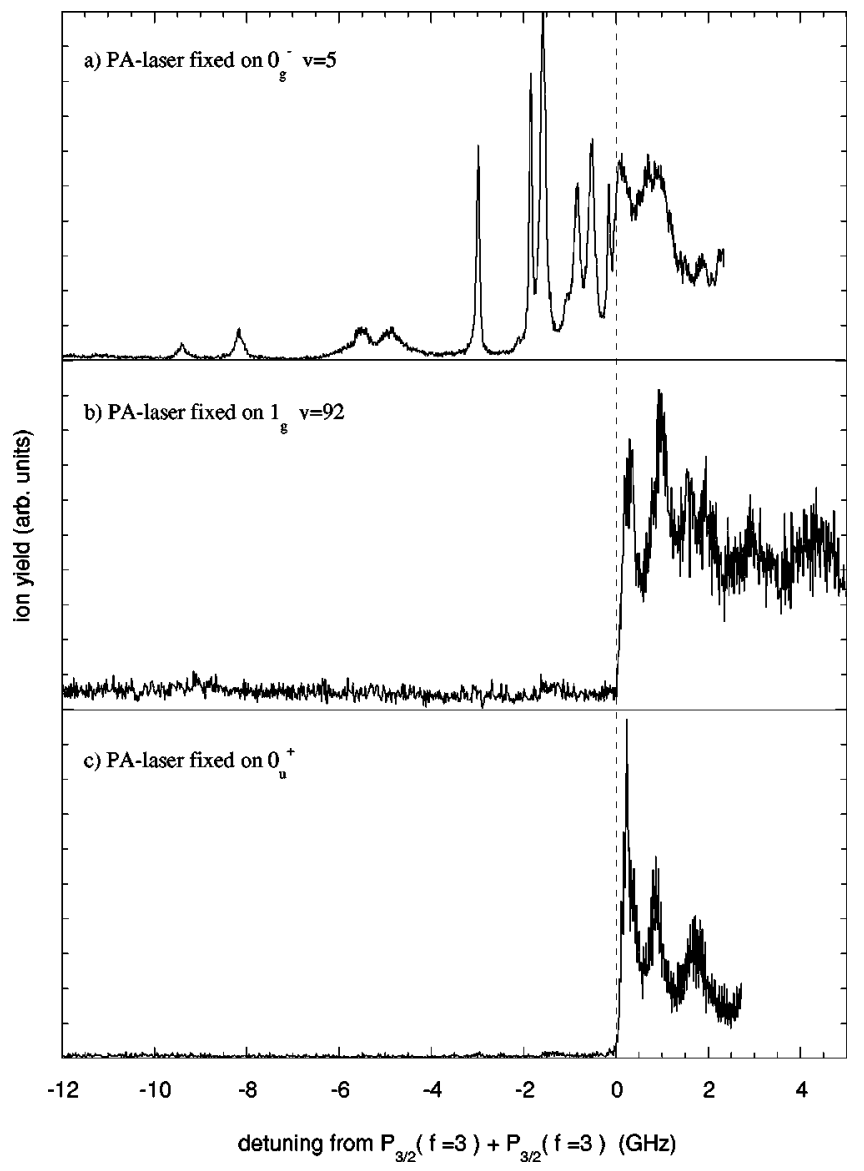

FIG. 6. Two-color measurement with the photoassociating (PA) laser fixed on the indicated vibrational states and the photoionizing laser scanned. Ion peaks below the dissociation limit are only observed with the PA laser fixed on a level in the $0_{g}^{-}$potential.

located near $50 a_{0}$ [7], whereas for both the $1_{g}$ and $0_{u}^{+}$vibrational levels the inner and outer turning points are located near $6 a_{0}$ and $130 a_{0}$, respectively. Thus the wave functions of these $1_{g}$ and $0_{u}^{+}$vibrational levels oscillate rapidly near $R=50 a_{0}$, resulting in a small Franck-Condon overlap with the doubly excited states at this internuclear distance. Unless the inner turning points of the doubly excited states match those of the intermediate states, the wave-function overlap at the inner turning points will also be negligible due to the rapid oscillations of both the intermediate and doubly excited state wave functions near their steep inner potential walls. Although the $1_{g}$ and $0_{u}^{+}$vibrational levels considered here are more deeply bound than those which will be populated by a laser detuned less than $5 \mathrm{GHz}$, the same reasoning holds for these higher levels since they have outer turning points at even larger distances than the levels considered here.

In contrast, the inner turning points of high vibrational levels of the long-range $0_{g}^{-}$potential are located near $55 a_{0}$. Figure 3 illustrates the situation. For these levels it is therefore reasonable to expect a good wave-function overlap with the least-bound doubly excited states. Our observation of doubly excited bound states shows that there is indeed a good wave-function overlap with the autoionizing doubly excited levels (which have been identified previously as levels

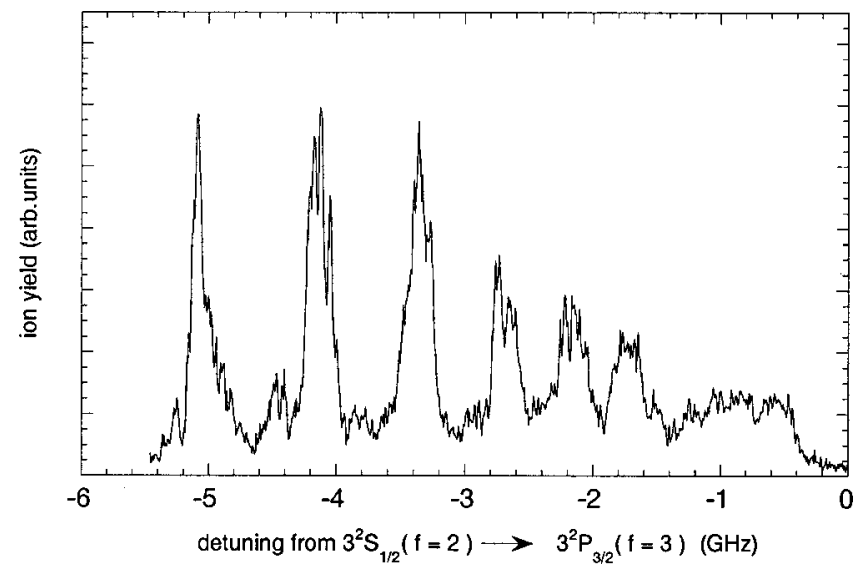

FIG. 7. Two-color measurement with the photoassociating laser scanning. The clearly resolved peaks are due to excitations to the $0_{g}^{-}(v=14-19)$ levels.

of $1_{u}$ and $0_{u}^{-}$symmetry [7]) at the inner turning point of the $0_{g}^{-}(v=5)$ level. Since the inner turning points of the $0_{g}^{-}$ levels do not change much with vibrational level, the vibrational levels important for the first $5 \mathrm{GHz}$ spectrum will have an overlap with the doubly excited states similar to that of the $v=5$ level used here. We have verified this experimentally for $v=9$.

\section{Two-color probe of the photoassociation step}

The observations and discussion of the preceding section confirm that the change in character of the single-color PAI spectrum for detunings less than $5 \mathrm{GHz}$ is due to a doubly resonant photoassociation process, as illustrated in Fig. 3. The first step is a free $\rightarrow$ bound transition to a high ( $v$ $\geqslant 12$ ) vibrational level of the $0_{g}^{-}$potential. The binding energies and widths of these high vibrational levels of the singly excited $0_{g}^{-}$state have been determined [8]. Figure 7 shows a measurement of these vibrational levels using the two-color ionization scheme as reported in Ref. [8] but with improved resolution. The two colors are related through $\nu_{\text {blue }}=\nu_{\text {red }}+28.5 \mathrm{GHz}$. Photoassociated singly excited molecules are promoted to a free doubly excited state (by absorption of a blue detuned photon $\nu_{\text {blue }}$ ) which autoionizes at short internuclear distances. The clearly resolved peaks are due to excitations to the $0_{g}^{-}(v=14-19)$ levels, while the bound states with binding energies smaller than $1.7 \mathrm{GHz}$ are broadened due to their predissociation into the $3 S_{1 / 2}(f=1)$ $+3 P_{3 / 2}$ dissociation continuum. Spectra of lower $0_{g}^{-}$vibrational levels [9] show a rotational progression with $J=2$ the dominant line, although $J=0$ through 4 are generally present. For the high vibrational levels shown here the rotational splittings are smaller than the hyperfine structure, so only broad lines are observed. We assume that the $J=2$ character still dominates. The spectrum of Fig. 7 was taken with a bright MOT and thus the dominant photoassociation lines originate from $2+2$ hyperfine ground-state collisions.

\section{ANALYSIS OF THE SINGLE-COLOR SPECTRUM}

\section{A. Line positions}

By combining the information of the first step [Fig. 7, also displayed in Fig. 8(a)] with the recently measured binding 


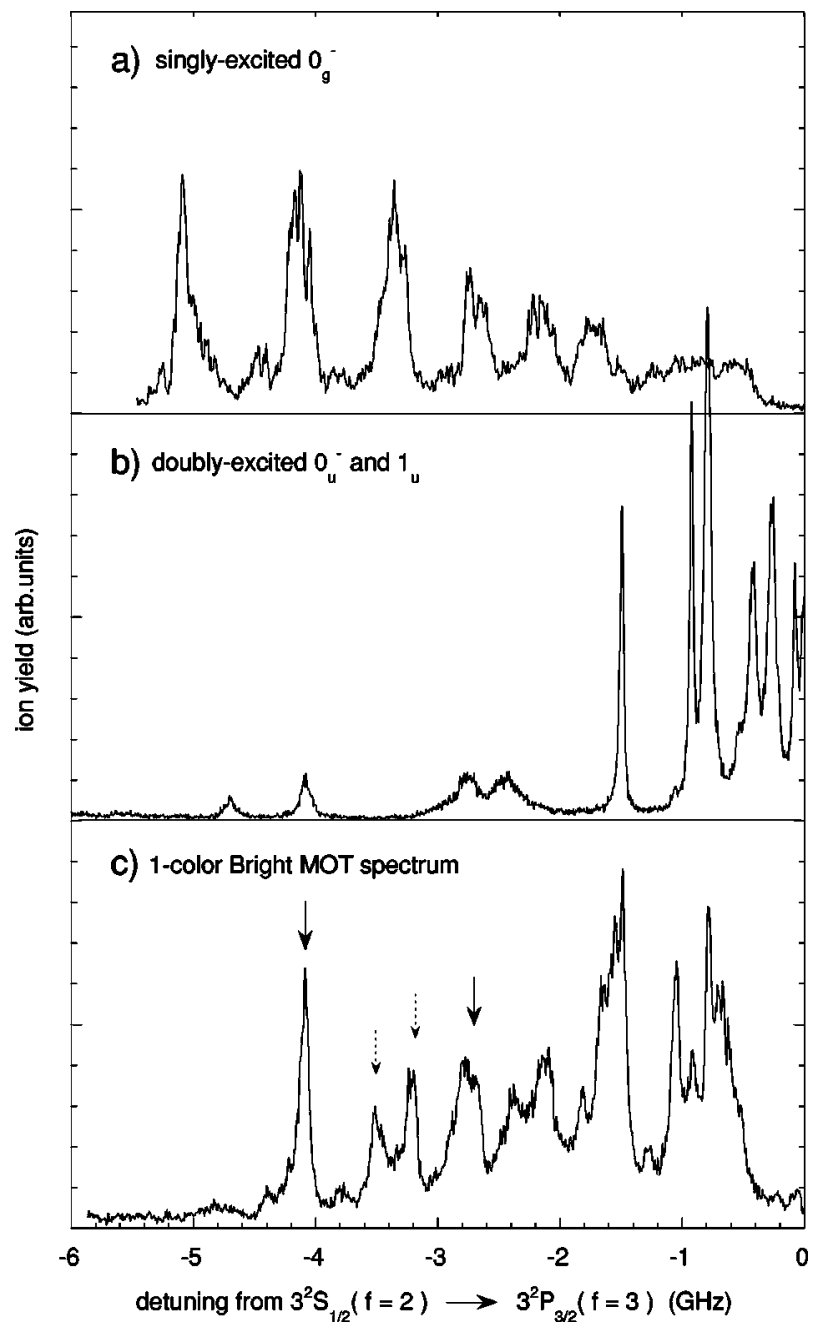

FIG. 8. A comparison of spectra used to identify lines in the single-color spectrum arising from $2+2$ ground state collisions. Panel (a) shows the singly excited $0_{g}^{-}$spectrum as a function of laser detuning taken in a bright MOT. Panel (b) shows the doubly excited bound-state spectrum as a function of laser detuning for 2 +2 collisions. The frequency scale is $1 / 2$ that of Fig. 6(a), as explained in the text. Panel (c) shows the one-color bright MOT spectrum. The solid arrows point to clear single-color double resonances, while the dotted arrows point to observed peaks which are not attributable to double resonances from the $2+2$ hyperfine ground state.

energies and widths of the doubly excited vibrational levels accessible from the $0_{g}^{-}$state as described in [7] and the preceding section [Fig. 6(a)], we can identify detunings for which there will be a single-color, doubly resonant process of photoassociation followed by excitation to an autoionizing level. Figure 8(b) shows the same spectrum as Fig. 6(a) but with the frequency scale divided by 2 . With this new scale, the figure shows the detunings for which two photons will have the correct total energy to produce a doubly excited state, starting with a pair of atoms colliding on the $2+2$ hyperfine ground state.

From this diagram we can see which features in the single-color bright MOT spectrum are attributable to photoassociation from the $2+2$ hyperfine ground state to a $0_{g}^{-}$line with $J=2$ character, followed by excitation to an autoionizing doubly resonant state. Two clear examples of such a double resonance are indicated by the solid arrows in Fig. 8(c). For example, the single-color bright MOT feature at $-4.1 \mathrm{GHz}$ is the result of a double resonance:

$$
\begin{aligned}
\mathrm{Na}(3 & S(f=2))+\mathrm{Na}(3 S(f=2))+2 h \nu \\
& \rightarrow \mathrm{Na}_{2}^{*}\left(0_{g}^{-}(v=15, J=2)\right)+h \nu \\
& \rightarrow \mathrm{Na}_{2}^{* *}\left(0_{u}^{-}\left(v=v_{\max }-1, J^{\prime}=3\right)\right) \rightarrow \mathrm{Na}_{2}{ }^{+}+e^{-},
\end{aligned}
$$

where the states are labeled according to Refs. [7] and [8]. Here $v_{\max }$ denotes the highest observed vibrational level of the doubly excited $0_{u}^{-}$state.

It can also be seen that some of the features in the bright MOT spectrum [specifically the structures at -3.25 and $-3.5 \mathrm{GHz}$, indicated by the dotted arrows in Fig. 8(c)] do not line up as nicely as the feature at $-4.1 \mathrm{GHz}$. This is mainly due to the fact that in a bright MOT some of the atoms are in the $f=1$ ground state. The alignment of Figs. 8 (a) and 8 (b) only applies to $2+2$ collisions. For $1+2$ collisions, Fig. 8(a) should be shifted to the right (blue) by 1.77 GHz while Fig. 8(b) should be shifted to the right by $1.77 / 2$ $\mathrm{GHz}$, as is shown in Fig. 9. These shifts will cause double resonances for $1+2$ collisions to occur for different laser detunings than for $2+2$ collisions, as can be seen in Fig. 9 . The solid arrow in Fig. 9(c) points at a clear double resonance from the $1+2$ hyperfine ground state, the presence of which cannot be understood by considering $2+2$ collisions only. Also not considered as yet are transitions in which the intermediate state has $J$ other than 2. For example, if the intermediate state has $J=3$ character, then the second step can be to the $J^{\prime}=4$ line of the doubly excited state; this has not been considered in our model.

\section{B. Peak heights}

In the preceding section we did not consider how the heights of the various peaks in the single-color spectra can be modeled. In fact, it is extremely difficult to calculate the spectrum of the first $5 \mathrm{GHz}$ ab initio, since that requires an accurate knowledge of the ground, singly excited, and doubly excited state wave functions, as well as all of the transition dipole moments. Here we present a more empirical model based on using measured two-color photoassociation spectra, which captures the main features of the experimental single-color spectra. We assume that the probability $P_{1}(\nu)$ for a transition from the $2+2$ ground state to the intermediate $0_{g}^{-}$state at a detuning $\nu$ is proportional to the ion yield for this detuning, as shown in Fig. 8(a). Likewise, the probability $P_{2}(\nu)$ for a transition from this intermediate state to the doubly excited states at that same detuning is proportional to the ion yield for this detuning, as shown in Fig. 8 (b). The contribution from $2+2$ collisions to the singlecolor spectra at a detuning $\nu$ can be obtained by multiplying the probabilities $P_{1}(\nu)$ and $P_{2}(\nu)$. Contributions from the $1+1$ and $1+2$ collisions are accounted for by shifting the spectra before multiplying, as discussed in the preceding section. The weights of the contributions from the different col- 


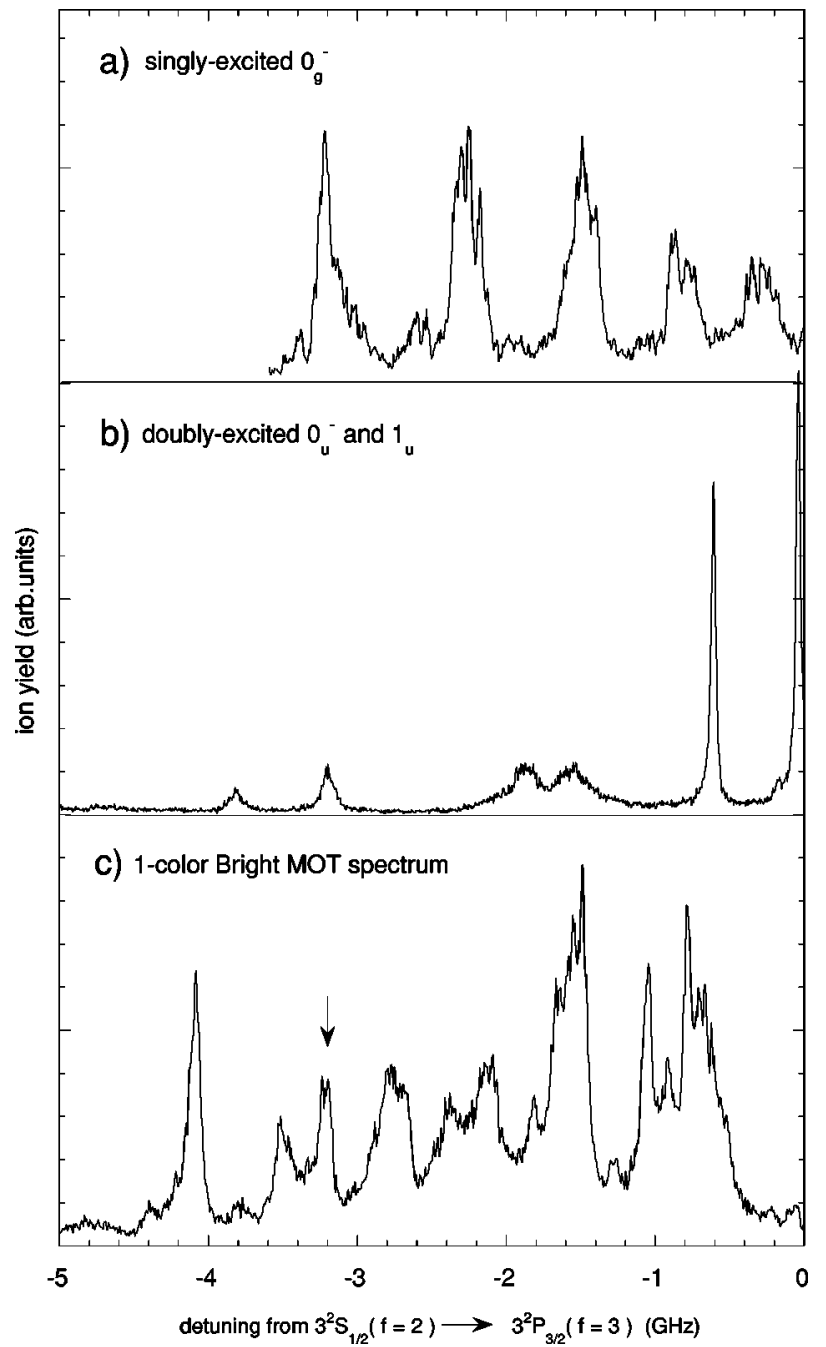

FIG. 9. A comparison of spectra used to identify lines in the single-color spectrum arising from $1+2$ ground-state collisions. Panel (a) shows the singly excited $0_{g}^{-}$spectrum as a function of laser detuning for $1+2$ collisions, panel (b) shows the doubly excited bound-state spectrum as a function of laser detuning for 1 +2 collisions, and panel (c) shows the one-color bright MOT spectrum. The solid arrow points to a clear double resonance from the $1+2$ hyperfine ground state.

lision states depend on the type of MOT used and are adjusted to reproduce the data. To account for the depletion of the MOT in the first $\approx 1.5 \mathrm{GHz}$ below atomic resonance caused by the near-resonant probe beam, we multiply the result by a Gaussian function fitted to the simultaneously recorded MOT-phase ion signal.

In Fig. 10 the calculated spectrum for the bright MOT is plotted together with the measurement. In the simulation the contribution from $1+2$ collisions is nine times smaller than that from $2+2$ collisions and with no contribution from 1 +1 collisions. All the calculated peaks show up also in the experimental spectrum. Two peaks in the experimental spectrum that are missing in the calculation are indicated by the arrows. This discrepancy between the calculation and the measurement is most likely caused by the assumption that only $J=2$ rotational levels are populated in the $0_{g}^{-}$state. The

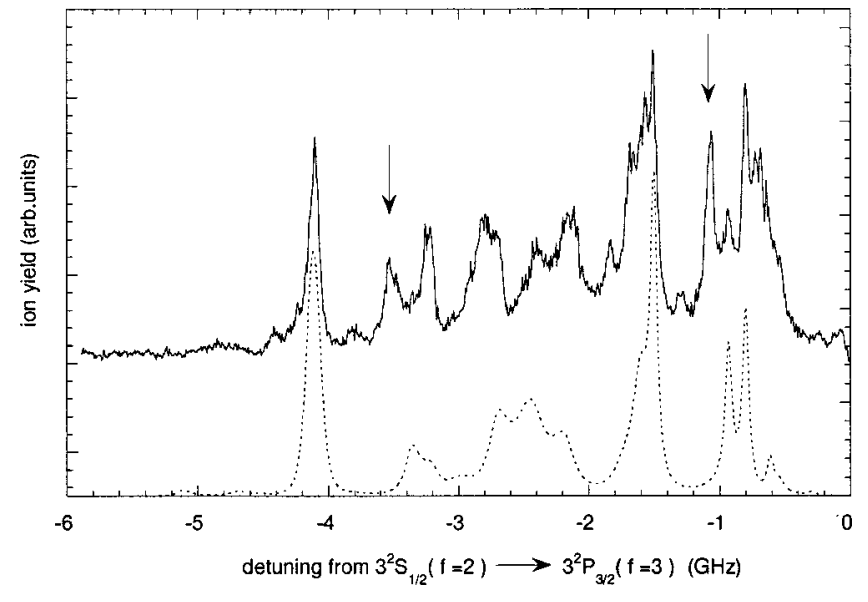

FIG. 10. Comparison between the calculated spectrum (dotted line) and the measurement (solid line) in the bright MOT. The origin of the lines indicated by arrows (which are missing in the calculation) is discussed in the text.

peak at $-3.5 \mathrm{GHz}$ can be attributed to a two-photon resonance from the $1+2$ hyperfine ground state to the doubly excited $0_{u}^{-}\left(v=v_{\max }-1, J^{\prime}=2\right)$ rotational level, while the peak at $-1.1 \mathrm{GHz}$ can be attributed to a two-photon resonance from the $2+2$ ground state to the doubly excited $0_{u}^{-}$ $\left(v=v_{\max }, J^{\prime}=2\right)$ level [7]. Since transitions from the singly excited $0_{g}^{-}(J=2)$ levels to the doubly excited $0_{u}^{-}\left(J^{\prime}=2\right)$ levels are forbidden [7], the $0_{u}^{-}\left(J^{\prime}=2\right)$ rotational levels do not appear in Fig. 8(b), and thus are also not included in the model spectrum of Fig. 10. In the experiment, however, the $0_{u}^{-}\left(J^{\prime}=2\right)$ levels may be excited from the intermediate $0_{g}^{-}$ $(J=1$ and $J=3)$ rotational levels, which may explain the discrepancy between the calculation and the measurement.

In Fig. 11 the calculated spectrum for the dark MOT is plotted together with the measurement. Again, there are no peaks predicted that do not appear in the experimental spectrum. In this case the contribution from $1+2$ collisions was weighted by a factor 0.5 relative to the dominant $1+1$ contribution, while no $2+2$ contribution was included. For the same reason as discussed for the bright MOT, the peak at

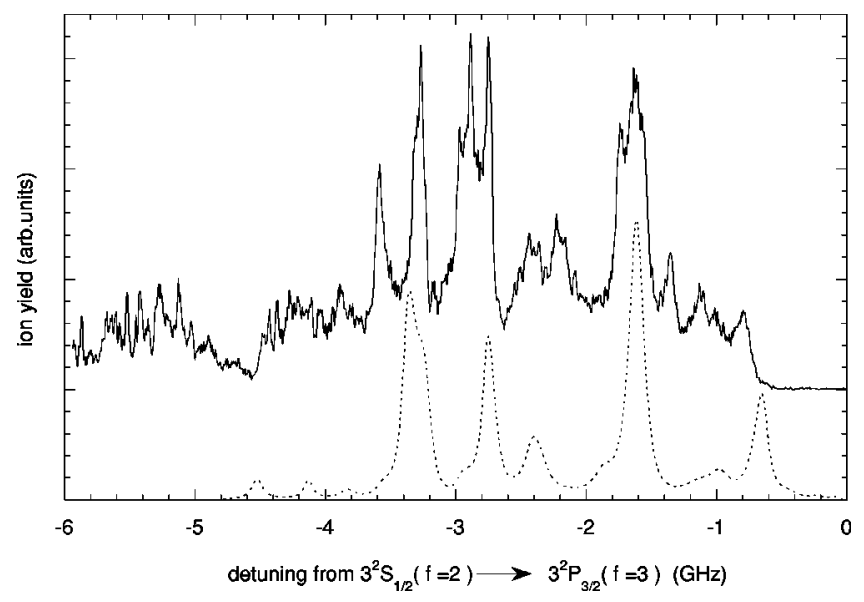

FIG. 11. Comparison between the calculated spectrum (dotted line) and the measurement (solid line) in the dark MOT. 
$-3.5 \mathrm{GHz}$ is missing in the calculation. Note that the peaks at -3.5 and $-3.25 \mathrm{GHz}$, which are present in both the bright and the dark MOT spectra, can be attributed to double resonances from the $1+2$ collision pair. [The peak at $-3.25 \mathrm{GHz}$ is a two-photon resonance from the $1+2$ ground state to the $0_{u}^{-}\left(v=v_{\max }-1, J^{\prime}=3\right)$ level.] This is reasonable, since the bright MOT spectrum is mainly a result of $2+2$ and $1+2$ collisions, whereas the dark MOT spectrum mainly results from $1+1$ and $1+2$ collisions. Similar peaks that appear in both the bright and dark MOT spectra are thus most likely a result of $1+2$ collisions.

We did not measure the relative populations of the $f=1$ and $f=2$ ground states in the bright and dark MOT configurations for these experiments. It is tempting to interpret the weighting factors for the contributions of $1+1,1+2$, and $2+2$ collisions as directly reflecting the populations of the different hyperfine collision states. Using the weighting factors in this way, however, ignores the fact that the spectrum originating from the $1+2$ initial state is not simply the spectrum from the $2+2$ state, shifted. In fact, the dipole moments from the different initial hyperfine ground states to the $0_{g}^{-}$state may differ, as do the Franck-Condon factors. Additionally, the experimental spectrum of Fig. 8(a) is not a direct measure of the photoassociation probability. The experiment uses a second, ionizing step [8] to detect that photoassociation has occurred. This second step is a bound $\rightarrow$ free transition that may impart some additional structure to the ionization signal [14]. An example of such a structure can be seen in the positive-detuning continua in Fig. 6. If we ignore these complications, we infer that in the bright MOT approximately $94 \%$ of the atoms are in the $f=2$ hyperfine ground state while in the dark MOT $75 \%$ of the atoms are in the $f=1$ state. In making this estimate, we have taken into account that the Bose symmetry of the colliding atoms limits the number of degenerate sublevels at the $1+1,1+2$, and $2+2$ asymptotes to 6,15 , and 15 , respectively, for $s$-wave scattering. We have further assumed that, on average, the transition probability from each of these sublevels to upper states is identical. The relative hyperfine state populations derived in this way are not unreasonable for our MOT conditions, suggesting that the other factors we have ignored do not, in aggregate, significantly modify the observed intensity ratios. The percentage of $f=1$ atoms in the dark MOT is low compared to values reported in [18], but under other circumstances we have produced higher ratios of $f=1$ to $f=2$ atoms in this apparatus as well.

\section{CONCLUSION}

In conclusion, we have shown that the intermediate $0_{g}^{-}$ state is the primary state involved in the ionization in the first $5 \mathrm{GHz}$ below the atomic resonance. The differences as well as the similarities between the bright and dark MOT spectra are explained by our model. The agreement between our model and the measurements shows that our model explains the radically different behavior of the single-color PAI spectrum in the first $5 \mathrm{GHz}$ from resonance in comparison with that at larger detunings.

\section{ACKNOWLEDGMENTS}

This work is part of the research program of the "Stichting voor Fundamenteel Onderzoek der Materie', (FOM), which is financially supported by the "Nederlandse Organisatie voor Wetenschappelijk Onderzoek" (NWO). This work was also supported by NIST and the U.S. Office of Naval Research.
[1] J. Weiner, V.S. Bagnato, S. Zilio, and P.S. Julienne, Rev. Mod. Phys. 71, 1 (1999).

[2] P.D. Lett, P.S. Jessen, W.D. Phillips, S.L. Rolston, C.I. Westbrook, and P.L. Gould, Phys. Rev. Lett. 67, 2139 (1991).

[3] L.P. Ratliff, M.E. Wagshul, P.D. Lett, S.L. Rolston, and W.D. Phillips, J. Chem. Phys. 101, 2638 (1994).

[4] P.D. Lett, K. Helmerson, W.D. Phillips, L.P. Ratliff, S.L. Rolston, and M.E. Wagshul, Phys. Rev. Lett. 71, 2200 (1993).

[5] J.J. Blangé, J.M. Zijlstra, A. Amelink, X. Urbain, H. Rudolph, P. van der Straten, H.C.W. Beijerinck, and H.G.M. Heideman, Phys. Rev. Lett. 78, 3089 (1997).

[6] R.W. Heather and P.S. Julienne, Phys. Rev. A 47, 1887 (1993).

[7] A. Amelink, K.M. Jones, P.D. Lett, P. van der Straten, and H. G.M. Heideman (unpublished).

[8] P.A. Molenaar, P. van der Straten, and H.G.M. Heideman, Phys. Rev. Lett. 77, 1460 (1996).

[9] K.M. Jones, P.S. Julienne, P.D. Lett, W.D. Phillips, E. Ties- inga, and C.J. Williams, Europhys. Lett. 35, 85 (1996).

[10] P. Kharchenko, J.F. Babb, and A. Dalgarno, Phys. Rev. A 55, 3566 (1997).

[11] M. Movre and G. Pichler, J. Phys. B 10, 2631 (1977).

[12] E. Tiesinga, C.J. Williams, P.S. Julienne, K.M. Jones, P.D. Lett, and W.D. Phillips, J. Res. Natl. Inst. Stand. Technol. 101, 505 (1996).

[13] W.C. Stwalley, Y. Uang, and G. Pichler, Phys. Rev. Lett. 41, 1164 (1978).

[14] K.M. Jones, S. Maleki, L.P. Ratliff, and P.D. Lett, J. Phys. B 30, 289 (1997).

[15] O. Dulieu, S. Magnier, and F. Masnou-Seeuws, Z. Phys. D 32, 229 (1994).

[16] E.L. Raab, M. Prentiss, A. Cable, S. Chu, and D.E. Pritchard, Phys. Rev. Lett. 59, 2631 (1987).

[17] W. Ketterle, K.B. Davis, M.A. Joffe, A. Martin, and D.E. Pritchard, Phys. Rev. Lett. 70, 2253 (1993).

[18] M. Mitsunaga and N. Imoto, Phys. Rev. A 59, 4773 (1999). 